\title{
Hazard Assessment of Iran Provinces based on the Health Ministry Tool in 2019
}

\author{
Ahmad Soltani ${ }^{1,2}$, Farshid Alaedini ${ }^{3}$, Navvab Shamspour ${ }^{3,4}$ and Milad Ahmadi Marzaleh ${ }^{2,5,6, *}$ \\ 1 Assistant Professor, Iran-Helal Institute, Red Crescent Society of the Islamic Republic of Iran, Tehran, Iran \\ 2 Research Center for Emergency and Disaster Resilience, Red Crescent Society of the Islamic Republic of Iran, Tehran, Iran \\ ${ }^{3}$ Research Center for Health Management in Mass Gathering, Red Crescent Society of the Islamic Republic of Iran, Tehran, Iran \\ ${ }^{4}$ Islamic Azad University, Tehran North Branch, Tehran, Iran \\ ${ }^{5}$ PhD of Health in Disasters and Emergencies, Student Research Committee, Department of Health in Disasters and Emergencies, Health \\ Human Resources Research Center, School of Management and Medical Informatics, Shiraz University of Medical Sciences, Shiraz, Iran \\ ${ }^{6}$ MPH of Health Policy, Health Policy Research Center, Institute of Health, Shiraz University of Medical Sciences, Fars, Iran
}

* Corresponding author: Milad Ahmadi Marzaleh, Research Center for Emergency and Disaster Resilience, Iran-Helal Institute of Applied Sciences and Technology, Red Crescent Society of the Islamic Republic of Iran, Tehran, Iran. Tel: +982188993959; Fax: +982188954007; Email: miladahmadimarzaleh@yahoo.com

Received 2020 November 03; Revised 2020 November 29; Accepted 2020 December 15.

\begin{abstract}
Background: Iran is a disaster-prone country, which is subjected to various hazards, such as floods, earthquakes, fire, and traffic accidents.

Objectives: This study aimed to determine the priority and risk of various hazards threatening the public in different provinces of Iran in 2019.

Methods: This cross-sectional study was carried out from March to September 2019 using data related to 31 provinces of Iran. The assessment was conducted using hazard assessment tools presented by the Iranian Ministry of Health. A total of 70 natural, man-made, and complex hazards were analyzed in this study. The data were collected by holding sessions and conducting individual and group interviews with the officials of provincial Red Crescent Societies as well as reviewing the databases of the Red Crescent Society and the Disaster Management Organization. The participants consisted of operation analysis experts.

Results: In terms of the frequency of occurrence, earthquake (12: 38.7\%), traffic accidents (7: 22.6\%), and flood (6: 19.4\%) obtained the highest priority in different provinces in descending order. Furthermore, regarding the total scores of hazards in all provinces, flood (78.6), earthquake (75.3), traffic accidents (71.9), drought (60.1), and building collapse (58.1) had the highest priority in descending order.

Conclusion: Given the extreme vulnerability of Iran to various disasters, authorities should develop strategic plans to reduce the risks associated with high-priority disasters. In addition, crisis and disaster management policymakers must develop separate detailed disaster response plans for each hazard in order to increase the preparedness at organizational and community levels. Public training can also raise awareness among the public and help people cope better with various hazards.
\end{abstract}

Keywords: Disaster, Hazard, Iran, Prioritization, Public training, Risk

\section{Background}

Natural disasters directly affect public health and result in injuries and deaths. They also increase physical and mental illnesses, disrupt social networks, and destroy physical territories and personal properties (1). Natural disasters (e.g., storms, floods, earthquakes, fire, drought, terrorist attack, volcanic eruption, and chemical disasters) and diseases may affect people in almost all countries. These disasters may start quickly or gradually. In any case, they have adverse effects on people, society, and the economy (2). Natural disasters are inevitable hazards that result in injuries, deaths, or destruction of buildings. About 771,911 people died and $1,917,557$ were affected by disasters worldwide between 2006 and 2015 (3).

With a population of about 80 million people, Iran is exposed to a wide range of natural and man-made hazards, such as earthquakes, drought, and traffic accidents $(4,5)$. Among about 300 most populated cities of Iran, approximately, $77 \%$ of them are built on faults and seismic zones and $50 \%$ are prone to floods (6). Furthermore, almost every 10 years, thousands of people are affected by large earthquakes with magnitudes greater than 7 Richter in Iran. In addition, people in some cities are exposed to storms and/or tidal waves. Therefore, these disasters lead to the death of about 5,000 people and loss of more than 100 billion Tomans each year (7). In total, 31 out of 40 known disasters occur in Iran (8). According to a report in 2016, Iran was ranked among the top 10 disaster-prone countries in the world. In the last 4 decades, about 109,000 Iranians have lost their lives due to disasters $(9,10)$. However, the frequency, severity, and outcome of disasters in Iran have increased in recent years (10).

Crises and disasters affect communities in many ways, including damage to humans, environment, and infrastructure. However, the vulnerability can be 
reduced through management measures, such as national and local capacity building, proper planning, and suitable training (11). Health centers, public societies, and relief organizations may be affected by crises and disasters, and therefore, are unable to help the survivors which per se can lead to secondary crises (12). Iran is a disaster-prone country, which is subject to various disasters, such as floods, earthquakes, fire, and traffic accidents.

\section{Objectives}

This study aimed to determine the priority and risk of various disasters threatening the general public in different provinces of Iran in 2019. Moreover, it was attempted to assess the need for developing public training programs.

\section{Methods}

This cross-sectional study was conducted from March to September 2019 using data related to 31 provinces of Iran. Hazard scores were calculated using a book entitled "Risk Assessment National Tools and Specialized Medical Surge Capacity Indicators in Disasters" written in 2014 for the Iranian Ministry of Health and Medical Education (13). A total of 70 natural, man-made, and complex hazards were analyzed in this study. The validity of the method has been confirmed by several Iranian crises and disaster experts and health managers. The risk scores were calculated using four indicators of the return period, intensity, vulnerability, and probability.

\subsection{Return period}

It refers to the recurrence intervals of a hazard in a geographical area. Hazards are classified into five levels based on their return periods (Table 1). A hazard, which has occurred repeatedly over the past 100 years, is placed in the fifth level. A return period obtains a coefficient of 2 . As shown in Table 1 , hazards are classified into five levels based on their return period.

\subsection{Intensity}

Hazard intensity is defined according to the number of people who died or were injured due to a hazard. The intensity of a hazard that has occurred frequently in an area is determined according to the number of people who died or were injured in the worst-case scenario. Hazard intensity has a coefficient of 5 . As can be seen in Table 2, hazards are classified into five levels based on their intensity.

\subsection{Vulnerability}

Vulnerability refers to the characteristics of a community that make it susceptible to the adverse effects of a hazard. Hazard vulnerability has a coefficient of 5. Hazards are classified into five levels based on the vulnerability of communities to their effects (Table 3).

\begin{tabular}{lcc}
\hline \multicolumn{2}{l}{ Table 1. Hazard classification based on return periods } & \\
\hline Level & Return period & Definition \\
\hline $\mathbf{1}$ & Very low & The hazard has not been recorded over the past 100 years. \\
$\mathbf{2}$ & Low & The hazard has occurred once over the past 100 years. \\
$\mathbf{3}$ & Medium & The hazard has occurred 2-3 times over the past 100 years. \\
$\mathbf{4}$ & High & The hazard has occurred 3-5 times over the past 100 years. \\
$\mathbf{5}$ & Very high & The hazard has occurred more than 5 times over the past 100 years. \\
\hline
\end{tabular}

\begin{tabular}{|c|c|c|}
\hline Level & Intensity & Definition \\
\hline 1 & Zero & The hazard has not affected the health of the public. \\
\hline 2 & Low & $\begin{array}{l}\text { Dead: } 1-2 \text { individuals } \\
\text { Injured: } 1-5 \text { individuals }\end{array}$ \\
\hline 3 & Medium & $\begin{array}{l}\text { Dead: } 3-5 \text { individuals } \\
\text { Injured: } 6-9 \text { individuals }\end{array}$ \\
\hline 4 & High & $\begin{array}{l}\text { Dead: 6-9 individuals } \\
\text { Injured: } 10-99 \text { individuals }\end{array}$ \\
\hline 5 & Very high & $\begin{array}{c}\text { Dead: } \geq 10 \text { individuals } \\
\text { Injured: } \geq 100 \text { individuals }\end{array}$ \\
\hline
\end{tabular}

Note: The fulfillment of one condition is sufficient, i.e., the intensity is determined based only on the number of injured cases or the number of deaths. The larger figure is taken into account. A hazard, which has killed 5 people and injured 100 individuals, is placed in the fifth level.

\begin{tabular}{lcc}
\hline \multicolumn{2}{l}{ Table 3. Hazard classification based on vulnerability } \\
\hline Level & Vulnerability & Definition \\
\hline $\mathbf{1}$ & Very low & Less than $20 \%$ of the population at risk may be physically, financially, or functionally affected. \\
$\mathbf{2}$ & Low & $20 \%-40 \%$ of the population at risk may be physically, financially, or functionally affected. \\
$\mathbf{3}$ & Medium & $40 \%-60 \%$ of the population at risk may be physically, financially, or functionally affected. \\
$\mathbf{4}$ & High & $60 \%-80 \%$ of the population at risk may be physically, financially, or functionally affected. \\
$\mathbf{5}$ & Very high & $80 \%-100 \%$ of the population at risk may be physically, financially, or functionally affected.
\end{tabular}




\begin{tabular}{lcc}
\hline \multicolumn{2}{l}{ Table 4. Hazard classification based on probability } \\
\hline Level & Probability & Definition \\
\hline $\mathbf{1}$ & Zero & The hazard never occurs in the desired area. \\
$\mathbf{2}$ & Low & The hazard may occur once over the next 75-100 years. \\
$\mathbf{3}$ & Medium & The hazard may occur once over the next 35-75 years. \\
$\mathbf{4}$ & High & The hazard may occur once over the next 5-35 years. \\
$\mathbf{5}$ & Very high & The hazard may occur once over the next 5 years. \\
\hline
\end{tabular}

\subsection{Probability}

Probability refers to the likelihood of the occurrence of a hazard at a specified time interval in the future. It is predicted through scientific observations or based on previous experiences and changes in the features of a geographical area. Probability has a coefficient of 7. According to Table 4 , hazards are classified into five levels based on their probability.

The coefficients of the return period (2), intensity (5), vulnerability (5) and probability (7) are constant. The final risk score (score range: 19-95) is calculated through the summation of the scores of these four indicators.

To calculate the risk level in each province, the score of each hazard in that province is multiplied by its population ratio. Subsequently, all hazard scores are summed and listed in descending order.

The data were collected by holding sessions and conducting individual and group interviews with the officials of provincial Red Crescent Societies and reviewing the databases of the Red Crescent Society and the Disaster Management Organization.

The participants consisted of operation analysis experts. Other data were collected from regional disaster management organizations, Iran Meteorological Organization, Geophysics Institute of the University of Tehran, provincial Red Crescent Societies, provincial fire departments, and local trustees. Finally, the data were analyzed in Excel 2016.

\section{Results}

Table 5 presents the priority and total risk scores of 11 frequent hazards in 31 provinces. Flood, earthquake, traffic accidents, and drought were generally the top priorities.

The frequency distribution of hazards with the highest priority in different provinces is shown in Figure 1. Earthquake, traffic accidents, and flood obtained the highest priority in descending order.

Table 6 tabulates the priority of 22 hazards based on the total hazard scores in all provinces.

\begin{tabular}{|c|c|c|c|c|c|c|c|c|c|c|c|c|}
\hline Hazard & & Earthquake & Flood & Landslide & $\begin{array}{l}\text { Rock-fall, } \\
\text { subsidenc, } \\
\text { quicksand }\end{array}$ & Storm & Tornado & Sandstorm & Dust & $\begin{array}{c}\text { Forest and } \\
\text { rangeland } \\
\text { fire }\end{array}$ & Drought & Terrorism \\
\hline East & Priority & 2 & 6 & 20 & & 13 & & & 7 & & 14 & \\
\hline Azerbaijan & Score & 90 & 73 & 35 & & 68 & & & 73 & & 67 & \\
\hline West & Priority & 7 & 4 & 12 & & 14 & & & & 13 & & \\
\hline Azerbaijan & Score & 49 & 55 & 33 & & 28 & & & & 29 & & \\
\hline \multirow{2}{*}{ Ardabil } & Priority & 13 & 1 & 23 & 14 & 20 & 19 & 44 & 15 & 7 & 17 & 32 \\
\hline & Score & 60 & 75 & 53 & 60 & 55 & 56 & 19 & 60 & 70 & 57 & 46 \\
\hline \multirow{2}{*}{ Esfahan } & Priority & 39 & 9 & 26 & 20 & 19 & 41 & 21 & 17 & 31 & 25 & 33 \\
\hline & Score & 40 & 90 & 63 & 65 & 70 & 19 & 65 & 70 & 56 & 64 & 51 \\
\hline \multirow{2}{*}{ Alborz } & Priority & 2 & 7 & 24 & 5 & 19 & & & & 18 & & \\
\hline & Score & 80 & 69 & 53 & 70 & 60 & & & & 61 & & \\
\hline \multirow{2}{*}{ Ilam } & Priority & 9 & 7 & 10 & & & & & 3 & 5 & & \\
\hline & Score & 53 & 63 & 46 & & & & & 75 & 65 & & \\
\hline \multirow{2}{*}{ Bushehr } & Priority & 1 & 2 & 22 & 23 & 16 & 20 & 24 & 14 & 25 & 13 & 26 \\
\hline & Score & 95 & 95 & 19 & 19 & 61 & 35 & 19 & 83 & 19 & 85 & 19 \\
\hline \multirow{2}{*}{ Tehran } & Priority & 4 & 1 & 23 & 24 & 19 & 38 & 20 & 21 & 26 & 28 & 14 \\
\hline & Score & 88 & 95 & 65 & 61 & 66 & 19 & 66 & 66 & 58 & 56 & 73 \\
\hline \multirow{2}{*}{$\begin{array}{l}\text { Chaharmah } \\
\text { al and } \\
\text { Bakhtiari }\end{array}$} & Priority & 4 & 3 & 5 & 6 & 13 & 19 & 23 & 16 & 10 & 32 & 37 \\
\hline & Score & 83 & 83 & 78 & 78 & 59 & 52 & 40 & 55 & 70 & 33 & 26 \\
\hline North & Priority & 1 & 3 & 11 & 8 & 12 & 29 & 33 & 23 & 9 & 30 & 31 \\
\hline Khorasan & Score & 83 & 75 & 37 & 42 & 36 & 17 & 19 & 28 & 42 & 19 & 19 \\
\hline Razavi & Priority & 7 & 4 & 12 & & 14 & & & & 13 & & \\
\hline Khorasan & Score & 49 & 55 & 33 & & 28 & & & & 29 & & \\
\hline South & Priority & 1 & 8 & 44 & 25 & 10 & 12 & 11 & 4 & 43 & 13 & 24 \\
\hline Khorasan & Score & 95 & 85 & 26 & 40 & 75 & 75 & 75 & 95 & 26 & 69 & 47 \\
\hline \multirow{2}{*}{ Khuzestan } & Priority & 3 & 1 & 32 & 17 & 28 & 29 & 30 & 2 & 25 & 42 & 8 \\
\hline & Score & 80 & 90 & 41 & 65 & 50 & 50 & 50 & 90 & 60 & 21 & 75 \\
\hline \multirow{2}{*}{ Zanjan } & Priority & 4 & 5 & 8 & 10 & 25 & 38 & 43 & 27 & 6 & 35 & 39 \\
\hline & Score & 80 & 80 & 73 & 75 & 54 & 26 & 19 & 49 & 80 & 30 & 24 \\
\hline \multirow{2}{*}{ Semnan } & Priority & 6 & 11 & 12 & 14 & 22 & 32 & 33 & 23 & 8 & & 34 \\
\hline & Score & 80 & 68 & 63 & 60 & 54 & 26 & 26 & 54 & 75 & & 26 \\
\hline \multirow{2}{*}{$\begin{array}{l}\text { Sistan and } \\
\text { Baluchestan }\end{array}$} & Priority & 1 & 4 & 28 & 31 & 9 & 43 & 11 & 2 & 19 & 40 & 3 \\
\hline & Score & 90 & 85 & 52 & 47 & 80 & 28 & 75 & 90 & 63 & 31 & 90 \\
\hline \multirow{2}{*}{ Fars } & Priority & 1 & 5 & 14 & 15 & 38 & 39 & 41 & 8 & 7 & 42 & 24 \\
\hline & Score & 95 & 90 & 73 & 73 & 26 & 26 & 19 & 76 & 80 & 19 & 52 \\
\hline \multirow{2}{*}{ Qom } & Priority & 18 & 17 & 35 & 16 & 34 & 45 & 37 & 38 & 39 & 7 & 8 \\
\hline & Score & 33 & 38 & 19 & 40 & 19 & 19 & 19 & 19 & 19 & 63 & 58 \\
\hline \multirow{2}{*}{ Qazvin } & Priority & 9 & 4 & & & 6 & & & & 7 & & \\
\hline & Score & 60 & 75 & & & 71 & & & & 63 & & \\
\hline
\end{tabular}




\begin{tabular}{|c|c|c|c|c|c|c|c|c|c|c|c|c|}
\hline \multirow{2}{*}{ Kurdistan } & Priority & 1 & 8 & 13 & 15 & 23 & 19 & 20 & 9 & 6 & 26 & 31 \\
\hline & Score & 95 & 80 & 73 & 60 & 45 & 45 & 45 & 80 & 85 & 35 & 28 \\
\hline \multirow{2}{*}{ Kerman } & Priority & 1 & 3 & 44 & 14 & 21 & 24 & 13 & 10 & 5 & 20 & 23 \\
\hline & Score & 95 & 90 & 19 & 73 & 51 & 46 & 73 & 78 & 85 & 55 & 50 \\
\hline \multirow{2}{*}{ Kermanshah } & Priority & 1 & 13 & 17 & 26 & 27 & 35 & 36 & 14 & 9 & 42 & 8 \\
\hline & Score & 95 & 80 & 75 & 70 & 70 & 49 & 49 & 80 & 85 & 45 & 85 \\
\hline \multirow{2}{*}{$\begin{array}{l}\text { Kohgiluyeh } \\
\text { and Boyer- } \\
\text { Ahmad }\end{array}$} & Priority & 11 & 1 & 15 & 12 & 30 & 31 & 32 & 16 & 23 & 9 & 33 \\
\hline & Score & 80 & 95 & 75 & 80 & 19 & 19 & 19 & 75 & 67 & 89 & 19 \\
\hline \multirow{2}{*}{ Golestan } & Priority & 1 & 4 & 12 & 32 & 31 & & 47 & 39 & & & \\
\hline & Score & 95 & 90 & 80 & 55 & 55 & & 19 & 19 & & & \\
\hline \multirow{2}{*}{ Gilan } & Priority & 3 & 1 & 9 & & 14 & & 43 & 34 & 13 & 27 & 31 \\
\hline & Score & 95 & 95 & 74 & & 63 & & 19 & 23 & 65 & 33 & 26 \\
\hline \multirow[b]{2}{*}{ Lorestan } & Priority & 1 & 5 & 9 & 23 & 13 & & & 4 & 8 & & \\
\hline & Score & 19 & 12 & 10 & 37 & 5 & & & 13 & 10 & & \\
\hline \multirow{2}{*}{ Mazandaran } & Priority & 4 & 1 & 5 & 6 & 8 & 39 & 40 & 33 & 10 & 44 & 41 \\
\hline & Score & 90 & 95 & 90 & 90 & 85 & 26 & 26 & 33 & 80 & 19 & 26 \\
\hline \multirow{2}{*}{ Markazi } & Priority & 1 & 2 & 4 & 17 & 16 & & & 6 & & & \\
\hline & Score & 78 & 78 & 77 & 65 & 71 & & & 76 & & & \\
\hline \multirow{2}{*}{ Hormozgan } & Priority & 6 & 4 & & 15 & 12 & & & 14 & 9 & & \\
\hline & Score & 74 & 77 & & 44 & 62 & & & 47 & 70 & & \\
\hline \multirow{2}{*}{ Hamedan } & Priority & 1 & 3 & 19 & 15 & 38 & 31 & 39 & 11 & 8 & 10 & 26 \\
\hline & Score & 85 & 80 & 53 & 60 & 19 & 19 & 19 & 65 & 70 & 68 & 40 \\
\hline \multirow{2}{*}{ Yazd } & Priority & & 7 & & & & & 3 & 5 & & & \\
\hline & Score & & 75 & & & & & 90 & 85 & & & \\
\hline
\end{tabular}

The frequency distribution of hazards with the highest priority in different provinces is shown in Figure 1. Earthquake, traffic accidents, and flood obtained the highest priority in descending order.

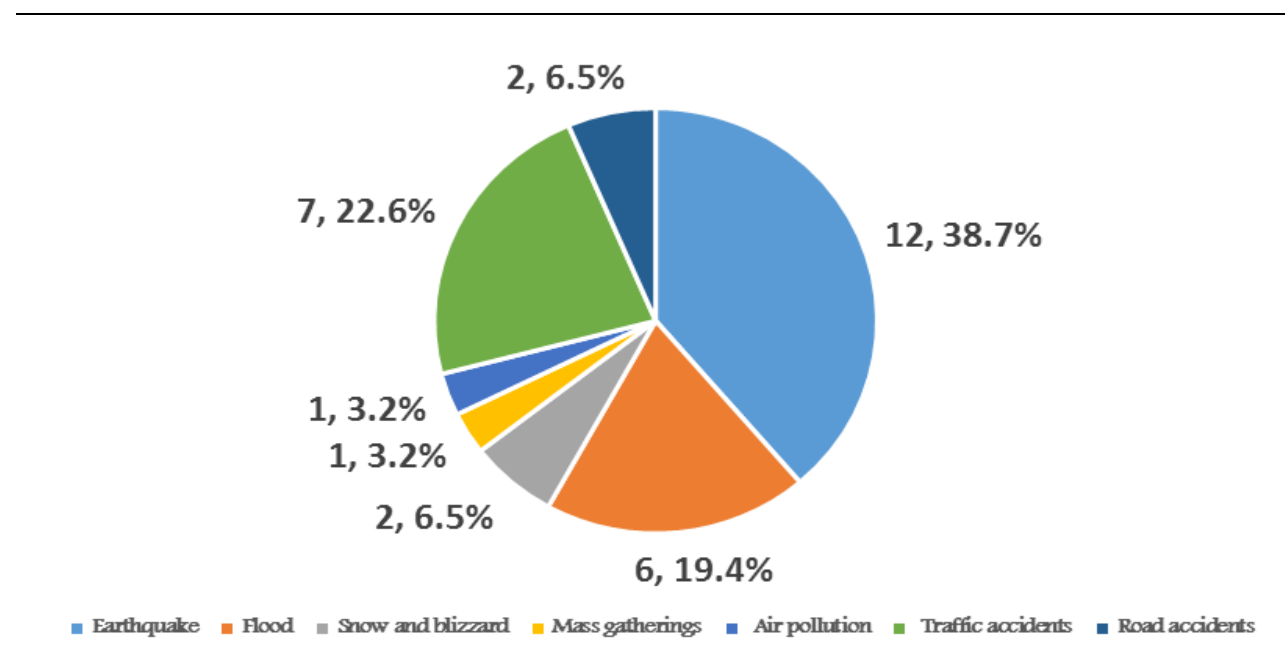

Figure 1. Frequency distribution of hazards with the highest priority in different provinces

\begin{tabular}{lccccc}
\hline \multicolumn{2}{l}{ Table 6. Priority of hazards based on total hazard scores } & & & \\
& & Total score & Priority & Hazard & Total score \\
\hline Priority & Hazard & 78.6 & $\mathbf{1 2}$ & Dust & 48.2 \\
\hline $\mathbf{1}$ & Flood & 75.3 & $\mathbf{1 3}$ & Storm & 48.0 \\
$\mathbf{2}$ & Earthquake & 71.9 & $\mathbf{1 4}$ & Air pollution & 47.1 \\
$\mathbf{3}$ & Traffic accidents & 60.1 & $\mathbf{1 5}$ & Rock-fall, subsidence, quicksand & 46.1 \\
$\mathbf{4}$ & Drought & 58.1 & $\mathbf{1 6}$ & Extremely high temperature & 43.9 \\
$\mathbf{5}$ & Building collapse & 57.9 & $\mathbf{1 7}$ & Railroad accidents & 42.6 \\
$\mathbf{6}$ & Snow and blizzard & 56.7 & $\mathbf{1 8}$ & Explosions and explosives & 42.4 \\
$\mathbf{7}$ & Industrial hazards & 56.3 & $\mathbf{1 9}$ & Aviation accidents & 39.4 \\
$\mathbf{8}$ & Building (structure) fire & 53.8 & $\mathbf{2 0}$ & Avalanche & 38.5 \\
$\mathbf{9}$ & Forest and rangeland fire & 50.5 & $\mathbf{2 1}$ & Beach accidents & 38.4 \\
$\mathbf{1 0}$ & Landslide & 50.4 & $\mathbf{2 2}$ & Epidemic diseases & 37.4 \\
$\mathbf{1 1}$ & Sandstorm & & & & \\
\hline
\end{tabular}

\section{Discussion}

This study aimed to analyze and prioritize various hazards in different provinces of Iran. Based on the results, earthquakes, traffic accidents, floods, and drought obtained the highest scores and priority; moreover, they showed occurrences in almost all provinces. Similar to this finding, the United Nations refers to drought as the most devastating natural disaster (14). In addition, $62 \%$ of the US lands were affected by a continental drought between 2011 and 2012. This drought exceeded $99 \%$ for drought size and affected about 150 million people (15).

According to a study conducted by Ardalan et al. 
(2013), damage to health facilities is the most important earthquake-related hazard in Iran. They also argued that structural damages account for $53.8 \%$ of all damages. This result is in line with the finding of the present study. Earthquake is a major cause of death among the health personnel and the most important cause of structural and nonstructural damage to primary health centers (4). The seasonal flood was the fifth most important hazard with a mean score of 45.96 (score range: 19-79). According to the 2015 World Disasters Report, the flood was among the most frequently reported disasters worldwide between 2005 and 2014. During this period, 1751 floods occurred throughout the world, which killed 59,092 people, injured 866,417 others, and imposed a significant burden of 342,836 million USD on the global economy (The 2015 World Disasters Report). In Thailand, the worst flood disaster in at least five decades occurred in 2011 with 1085 deaths and an economic loss of about 45.7 billion USD. This was the fourth costliest natural disaster in recent history (16).

Hospital Safety Index is among the most common tools used by researchers for assessing the disaster risk in hospitals. It can also be used to assess the risks threatening the communities. In Iran, researchers have drawn hazard maps to assess the vulnerability of Kerman and Tehran provinces to natural hazards. The effectiveness of risk assessment depends on organizational commitment and inter-organizational collaboration at high levels (17).

In total, 119 natural disasters were recorded in 25 provinces of Iran (11.9 events per year) between 2001 and 2011. The disasters threatened the lives and safety of many health staff who worked in primary health care centers in the affected cities. These events led to the physical damage or functional failure in 1401 health centers, injury or illness of 644 people, and death of 127 health staff. The most adverse effects of the disasters were observed in the health centers of Kerman, Sistan and Baluchestan, and Lorestan (18). More than 100 hospitals and 650 health centers were affected by natural disasters worldwide between 1990 and 2010; moreover, patients and staff were evacuated from many hospitals due to their unsafe conditions (19).

Risk analysis is a stable approach; therefore, relevant analyses and assessments should be performed at short intervals. In addition, low-priority hazards should also be taken into consideration when developing risk-reduction plans. Disaster risk assessment can help disaster decision-makers allocate adequate resources and budgets to relevant programs. Regarding the limitations of this study, there was no comprehensive database of information about disasters, which occurred in the last decade in Iran. In addition, despite the long return period of some disasters, the researchers only used available news archives and some local sources of information to examine past events.

With this background in mind, further studies are recommended to analyze various hazards on an annual basis, use different risk analysis approaches, get highly-qualified experts involved in the analysis process, promote resilience to various hazards, and take necessary measures to reduce the risks and increase relevant capacities.

\section{Conclusion}

Given the extreme vulnerability of Iran to various disasters, authorities should develop strategic plans to reduce the risks associated with high-priority disasters. The results demonstrate the need for a comprehensive general and specialized training program to prepare people for floods, earthquakes, and road accidents. In addition, crisis and disaster management policymakers must develop separate detailed disaster response plans for each hazard in order to increase the preparedness at organizational and community levels. Public training can also raise awareness among the public and help people better cope with various hazards.

\section{Acknowledgments}

The authors express their gratitude to the administration and personnel of the Iranian Red Crescent Society for paving the way for conducting this study.

\section{Footnotes}

Authors' Contribution: Ahmad Soltani, Farshid Alaedini, Navvab Shamspour, and Milad Ahmadi Marzaleh were responsible for the study conception and design. Ahmad Soltani, Farshid Alaedini, Navvab Shamspour, and Milad Ahmadi Marzaleh supervised the whole thesis. Ahmad Soltani, Farshid Alaedini, Navvab Shamspour, and Milad Ahmadi Marzaleh prepared the first draft of the manuscript. Ahmad Soltani, Farshid Alaedini, Navvab Shamspour, and Milad Ahmadi Marzaleh performed the analysis of the results and supervised the study. All authors have read and approved the final manuscript.

Conflict of Interests: The authors have no conflict of interest to declare.

Funding/Support: This project has partly been supported by a grant from the Iranian Red Crescent Society.

Financial Disclosure: None declared. Informed consent: Nil.

\section{References}

1. Pan American Health Organization. Natural disasters: protecting the public's health. Washington, D.C: Pan American Health Organization; 2000.

2. Guha-Sapir D, Vos F, Below R, Ponserre S. Annual disaster 
statistical review 2011: the numbers and trends. Brussels, Belgium: Centre for Research on the Epidemiology of Disasters (CRED); 2012.

3. Sanderson D, Sharma A. World disasters report 2016. Resilience: saving lives today, investing for tomorrow. Geneva, Switzerland: International Federation of Red Cross and Red Crescent Societies; 2016.

4. Ardalan A, Mowafi H, Homa Yousefi K. Impacts of natural hazards on primary health care facilities of Iran: a 10-year retrospective survey. PLoS Curr. 2013;5:ecurrents.dis. ccdbd870f5d1697e4edee5eda12c5ae6. doi: 10.1371/currents. dis.ccdbd870f5d1697e4edee5eda12c5ae6. [PubMed: 23863871].

5. Azami-Aghdash S, Gorji HA, Sadeghi-Bazargani H, Shabaninejad H. Epidemiology of road traffic injuries in Iran: based on the data from Disaster Management Information System (DMIS) of the Iranian Red Crescent. Iran Red Crescent Med J. 2017;19(1):e38743. doi: 10.5812/ircmj.38743.

6. Dashtpagerdi MM, Kousari MR, Vagharfard H, Ghonchepour D, Hosseini ME, Ahani H. An investigation of drought magnitude trend during 1975-2005 in arid and semi-arid regions of Iran. Environ Earth Sci. 2015;73(3):1231-44. doi: 10.1007/s12665014-3477-1.

7. Izadkhah Y, Amini Hosseini K. An evaluation of disaster preparedness in four major earthquakes in Iran. J Seismol Earthquake Eng. 2010;12(1-2):61-75.

8. Jahanbakhsh M, Tavakoli N, Hadadpour A. Designing disaster victims'medical record; a step toward crisis management. Health Inform Manag. 2011;7(4):400-9.

9. Ardalan A, Kandi M, Osooli M, Shamseddini A. Profile of natural hazards in IR Iran, 1970-2010. Tehran, Iran: National Institute of Health Research; 2012.

10. Ager A, Baillie Smith M, Barbelet V, Carpenter S, Carter W, Cartwright A, et al. World disasters report: focus on local actors, the key to humanitarian effectiveness. Geneva, Switzerland: International Federation of Red Cross and Red
Crescent Societies; 2015.

11. United Nations International Strategy for Disaster Reduction. Hyogo framework for action 2005-2015: building the resilience of nations and communities to disasters. Extract from the final report of the World Conference on Disaster Reduction (A/CONF 206/6). Geneva, Switzerland: United Nations International Strategy for Disaster Reduction; 2005.

12. Hospitals Safe From Disasters. 2009 world disaster reduction campaign. Reduce risk, protect health facilities, save lives. Californi: United Nations; 2009.

13. Khankeh H. Collection of national tools for risk assessment and indicators of specialized health capability in accidents and disasters (Hazard Map). Tehran, Iran: University of Social Welfare and Rehabilitation Sciences; 2015.

14. Hayes M, Svoboda M, Wall N, Widhalm M. The Lincoln declaration on drought indices: universal meteorological drought index recommended. Bull Am Meteoroll Soc. 2011;92(4):485-8. doi: 10.1175/2010BAMS3103.1.

15. Cook BI, Smerdon JE, Seager R, Cook ER. Pan-continental droughts in North America over the last millennium. J Climate. 2014;27(1):383-97. doi: 10.1175/JCLI-D-13-00100.1.

16. Benfield A. Thailand floods event recap report. Chicago: Aon Benfield Analytics; 2011.

17. Ardalan A, Keleh MK, Saberinia A, Khorasani-Zavareh D, Khankeh H, Miadfar J, et al. 2015 estimation of hospitals safety from disasters in IR Iran: the results from the assessment of 421 hospitals. PloS One. 2016;11(9):e0161542. doi: 10.1371/journal.pone.0161542. [PubMed: 27602564].

18. Salamati Nia S, Kulatunga U. The importance of disaster management and impact of natural disasters on hospitals. The 6th World Construction Symposium, Sri Lanka; 2017.

19. Bosher L, Dainty A. Disaster risk reduction and 'built-in'resilience: towards overarching principles for construction practice. Disasters. 2011;35(1):1-18. doi: 10.1111/j.1467-7717.2010.01189.x. [PubMed: 20722695]. 